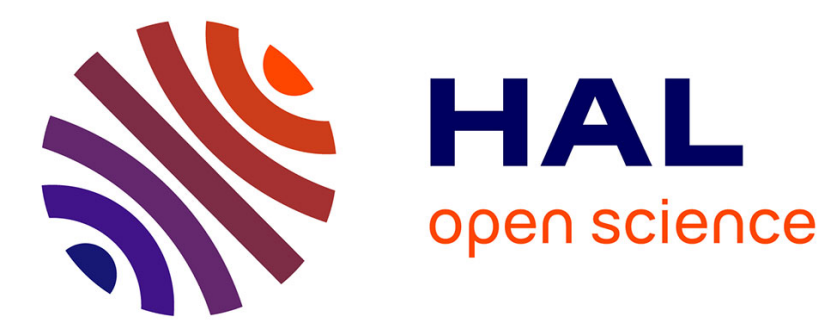

\title{
Inference of curvature using tubular neighborhoods
} Frédéric Chazal, David Cohen-Steiner, André Lieutier, Quentin Merigot, Boris Thibert

\section{To cite this version:}

Frédéric Chazal, David Cohen-Steiner, André Lieutier, Quentin Merigot, Boris Thibert. Inference of curvature using tubular neighborhoods. L. Najman; P. Romon. Lecture Notes in Mathematics, 2184, Springer, pp.133-158, 2017, Modern Approaches to Discrete Curvature, 978-3-319-58001-2. 10.1007/978-3-319-58002-9_4 . hal-01425558

\section{HAL Id: hal-01425558 https://hal.science/hal-01425558}

Submitted on 3 Jan 2017

HAL is a multi-disciplinary open access archive for the deposit and dissemination of scientific research documents, whether they are published or not. The documents may come from teaching and research institutions in France or abroad, or from public or private research centers.
L'archive ouverte pluridisciplinaire HAL, est destinée au dépôt et à la diffusion de documents scientifiques de niveau recherche, publiés ou non, émanant des établissements d'enseignement et de recherche français ou étrangers, des laboratoires publics ou privés. 


\title{
Inference of curvature using tubular neighborhoods
}

\author{
F. Chazal D. Cohen-Steiner A. Lieutier Q. Mérigot \\ B. Thibert
}

December 31, 2016

\section{Introduction}

Geometric inference deals with the problem of recovering the geometry and topology of a compact subset $K$ of $\mathbb{R}^{d}$ from an approximation by a finite set $P$. This problem has seen several important developments in the previous decade. Many of the proposed constructions share a common feature: they estimate the geometry of the underlying compact set $K$ using offsets of $P$,

$$
P^{r}:=\bigcup_{p \in P} \mathrm{~B}(p, r)
$$

which can also be seen as the $r$-sublevel set of the distance function to $P$. These offset correspond to what is called tubular neighborhoods in differential geometry. First and second-order geometric quantities are encoded in the tube $K^{r}$ around a manifold. For instance, the classical tube formula asserts that it is possible to estimate the curvature of a compact smooth submanifold $K$ from the volume of its offsets. One can hope that if the finite set $P$ is close to $K$ in the Hausdorff sense, some of this geometric information remains in the offsets of $P$. In this chapter, we will see how this idea can be used to infer generalized notions of curvature such as Federer's curvature measures.

Notation The space $\mathbb{R}^{d}$ is equipped with the canonical dot product $\langle. \mid$. and the induced norm $\|\cdot\|$

\section{Distance function and sets with positive reach}

Throughout this chapter, $K$ will denote a compact set in the Euclidean $d$ space $\mathbb{R}^{d}$, usually with no additional regularity assumption. The distance 
function to $K$, denoted $\mathrm{d}_{K}$, is defined by

$$
\mathrm{d}_{K}: x \in \mathbb{R}^{d} \mapsto \min _{p \in K}\|x-p\|
$$

In this short section, we review some regularity properties of the distance function to a compact set, which we will use to establish stability results for generalized notions of curvature. We also introduce the class of sets with positive reach and sets with positive $\mu$-reach, for which it is possible to define and infer generalized notions of curvature.

Definition 1 (Offset). The $r$-offset of $K$, also called tubular neighborhood in geometry, is the set $K^{r}$ of points at distance at most $r$ of $K$, or equivalently the sublevel set $K^{r}:=\left\{x \in \mathbb{R}^{d} ; \mathrm{d}_{K}(x) \leq r\right\}$.

Definition 2 (Hausdorff distance). The Hausdorff distance between two compact subsets $K$ and $P$ of $\mathbb{R}^{d}$ can be defined in term of offsets:

$$
\mathrm{d}_{\mathrm{H}}(K, P):=\min \left\{r \geq 0 \text { s.t. } K \subseteq P^{r} \text { and } P \subseteq K^{r}\right\}
$$

Loosely speaking, a finite set $P$ is within Hausdorff distance $r$ from a compact set $K$ if it is sampled close to $K\left(P \subseteq K^{r}\right)$ and densely in $K\left(K \subseteq P^{r}\right)$. An alternative characterization of the Hausdorff distance is given by the following equality, where $\|f\|_{\infty}=\sup _{x \in \mathbb{R}^{d}}|f(x)|$.

$$
\mathrm{d}_{\mathrm{H}}\left(K, K^{\prime}\right):=\left\|\mathrm{d}_{K}-\mathrm{d}_{K^{\prime}}\right\|_{\infty}
$$

\section{Gradient of the distance and sets with positive reach}

Projection function, medial axis and gradient The semi-concavity of the squared distance function to a compact set has been remarked and used in different contexts $[14,22,3,18]$. We will use the fact that for any compact subset $K \subseteq \mathbb{R}^{d}$, the square of the distance function to $K$ is 1 -semiconcave.

Definition 3. A function $\phi$ defined on a subset $\Omega$ of $\mathbb{R}^{d}$ is $\lambda$-concave if and only if the function $\phi-\lambda\|\cdot\|^{2}$ is concave.

It is easy to see that the distance function to a compact set is 1-Lipschitz. By Rademacher's theorem, this implies that this function is differentiable almost everywhere. The next proposition shows that the squared distance function to a compact set has the same regularity as a concave (or convex) function. In particular, Alexandrov's theorem implies that distance functions to compact set are twice differentiable almost everywhere. 
Definition 4 (Projection function and medial axis). A point $p$ of $K$ that realizes the minimum in the definition (2) of the distance function $\mathrm{d}_{K}(x)$ is called a projection of $x$ on $K$. The set of such projections is denoted $\operatorname{proj}_{K}(x)$, and is always non-empty by compactness of $K$. The medial axis of $K$, denoted $\operatorname{Med}(K)$ is the set of points $x$ in $\mathbb{R}^{d}$ that have more than one projection on $K$. On the complement of the medial axis, points have a single projection on $K$, allowing one to define a map $\mathrm{p}_{K}: \mathbb{R}^{d} \backslash \operatorname{Med}(K) \rightarrow K$ called the projection function on $K$.

Proposition 5. The squared distance function to a compact subset $K$ of $\mathbb{R}^{d}$ is 1-semiconcave and differentiable on $\mathbb{R}^{d} \backslash \operatorname{Med}(K)$. For every $x \notin$ $\operatorname{Med}(K) \cup K$, one has

$$
\nabla_{x} \mathrm{~d}_{K}^{2}=2\left(x-\mathrm{p}_{K}(x)\right) \quad \nabla_{x} \mathrm{~d}_{K}=\frac{x-\mathrm{p}_{K}(x)}{\left\|x-\mathrm{p}_{K}(x)\right\|}
$$

Proof. The function $\mathrm{d}_{K}(.)^{2}-\|\cdot\|^{2}$ is a minimum of linear functions:

$$
\begin{aligned}
\mathrm{d}_{K}(x)^{2}-\|x\|^{2} & =\min _{p \in P}\|x-p\|^{2}-\|x\|^{2} \\
& =\min _{p \in P}\|p\|^{2}-2\langle x \mid p\rangle
\end{aligned}
$$

and is therefore concave. A concave function is differentiable almost everywhere, and one has $\nabla_{x}\left[\mathrm{~d}_{K}(.)^{2}-\|\cdot\|^{2}\right]=-2 \mathrm{p}_{K}(x)$ at points of differentiability. This implies the desired formulas.

Sets with positive reach In his seminal article on curvature measures [12], Federer introduced the class of sets with positive reach, a class which generalizes both convex subsets and compact smooth submanifolds of $\mathbb{R}^{d}$.

Definition 6 (Reach). The reach of a compact set $K$, denoted by reach $(K)$ is the minimum distance between $K$ and its medial axis.

EXAMPLE 7. It is well known that the projection to a closed convex set $K \subseteq \mathbb{R}^{d}$ is uniquely defined on the whole space, so that reach $(K)=+\infty$. The reciprocal of this statement is a theorem of Motzkin [21]: if reach $(K)=$ $+\infty$, then $K$ is convex.

EXAMPLE 8. The tubular neighborhood theorem implies that a smooth compact submanifold of $\mathbb{R}^{d}$ always has positive reach. In the case of a smooth compact hypersurface, this follows from the proof of Proposition 23. In addition, the reach of submanifold $M$ is always less than the minimum radius of curvature of $M$. 
ExAmple 9. Note however, that the reach is a global quantity, and cannot be lower bounded by any function of the minimum curvature radius. For instance, consider a compact set consisting of two spheres of radius $R$ at distance $\varepsilon$. Then, the reach of the union of those two sphere is $\frac{\varepsilon}{2}$ while the minimum curvature radius remains constant and equal to $R$. It is also possible to construct similar examples involving connected manifolds.

The definition of curvature measures of sets with positive reach relies on the fact that the boundary of small tubular neighborhoods around those sets are hypersurface of class $\mathcal{C}^{1,1}$, that is $\mathcal{C}^{1}$ hypersurface with a Lipschitz normal vector field (see [12, Theorem 4.8]).

Proposition 10 ([12]). Let $K \subseteq \mathbb{R}^{d}$ be a set with positive reach. Then, for any $r$ in $(0, \operatorname{reach}(K))$ the restriction of the projection function to $K^{r}$ is Lipschitz. In particular, the level set $\partial K^{r}=\mathrm{d}_{K}^{-1}(\{r\})$ is a $\mathcal{C}^{1,1}$ hypersurface.

\section{Generalized gradient and sets with positive $\mu$-reach}

The reach of a compact set a very unstable quantity. For instance, the reach of a triangulation is always zero, whereas smooth surfaces, which have positive reach, can be approximated arbitrarily well by triangulations. In this section, we will see how to define a relaxed notion of reach using a generalized gradient of the distance function.

Generalized gradient The distance function to a compact set is differentiable everywhere but on the medial axis. However, the semiconcavity property allows one to define a generalized gradient of the distance function everywhere. This generalized gradient coincides with the usual gradient of the distance function when it is differentiable. ${ }^{1}$

Definition 11. Let $K$ be a compact subset of $\mathbb{R}^{d}$ and let $x$ be a point in $\mathbb{R}^{d} \backslash K$. We define the generalized gradient of the distance function to $K$ at $x$ by:

$$
\nabla \mathrm{d}_{K}(x)=\frac{x-\widetilde{p}_{K}(x)}{\mathrm{d}_{K}(x)}
$$

where $\widetilde{p}_{K}(x)$ is the center of the (uniquely defined) smallest ball containing the set of projections $\operatorname{proj}_{K}(x)$.

\footnotetext{
${ }^{1}$ This generalized gradient coincides with the orthogonal projection of the origin on the supdifferential of the distance function [4, Lemma 5.2].
} 
In particular, the norm $\left\|\nabla \mathrm{d}_{K}(x)\right\|$ equals one if and only if $\operatorname{proj}_{K}(x)$ is contained in a ball with zero radius, i.e. if it is a singleton. In other words,

$$
\operatorname{Med}(K)=\left\{x \in \mathbb{R}^{d} \backslash K ;\left\|\nabla \mathrm{d}_{K}(x)\right\|<1\right\} .
$$

Sets with positive $\mu$-reach. The $\mu$-reach is a relaxed version of the reach, which had originally been introduced because of its better stability properties with respect to perturbations of the compact set [5]. By Equation (7), the reach of a compact set $K$ is equal to the maximal radius $r$ such that $\left\|\nabla \mathrm{d}_{K}(x)\right\|=1$ on the offset $K^{r}$ :

$$
\operatorname{reach}(K)=\sup \left\{r \geq 0 ; \forall x \in K^{r},\left\|\nabla \mathrm{d}_{K}(x)\right\|=1\right\} .
$$

This remark suggests a parameterized notion of reach, called the $\mu$-reach.

Definition 12 ( $\mu$-Reach). Let $\mu \in(0,1)$. The $\mu$-reach of a compact set $K$ is the maximal radius $r$ such that $\left\|\nabla \mathrm{d}_{K}(x)\right\| \geq \mu$ on the offset $K^{r}$ :

$$
\operatorname{reach}_{\mu}(K)=\sup \left\{r \geq 0 ; \forall x \in K^{r},\left\|\nabla \mathrm{d}_{K}(x)\right\| \geq \mu\right\} .
$$

With $\mu=1$, we recover the notion of reach introduced earlier.

In addition to smooth manifolds and convex sets, the class of compact sets with positive $\mu$-reach also contains triangulations and non-convex polyhedra. Offsets of sets with positive $\mu$-reach are not smooth in the sense of Proposition 10, but they still possess some regularity properties.

Offsets of compact sets with positive $\mu$-reach Let $K \subset \mathbb{R}^{d}$ be a compact set with positive $\mu$-reach. A theorem of $\mathrm{Fu}[15$, Corollary 3.4] implies that for any radius $r$ in $\left(0, \operatorname{reach}_{\mu}(K)\right)$, the closure of the complement of the tubular neighborhood $K^{r}$ has positive reach. This lower bound was made quantitative in [6].

Theorem 13 ([6]). Let $K \subseteq \mathbb{R}^{d}$ be a set with positive $\mu$-reach. Then, for any radius $r$ in $\left(0, \operatorname{reach}_{\mu}(K)\right)$, one has

$$
\operatorname{reach}\left(\overline{\mathbb{R}^{d} \backslash K^{r}}\right) \geq \mu r .
$$

From Proposition 10, this implies that offsets of $\overline{\mathbb{R}^{d} \backslash K^{r}}$ are of class $\mathcal{C}^{1,1}$. These so-called double offsets are used in computer aided design to 
smoothen a surface. The $(r, t)$-double offset of $K$ is the set of points that are at distance $t$ of the complementary of $K^{r}$ :

$$
K^{r, t}:={\overline{\mathbb{R}^{d} \backslash K^{r}}}^{t}=\left\{x \in \mathbb{R}^{d} ; \mathrm{d}\left(x, \overline{\mathbb{R}^{d} \backslash K^{r}}\right) \leq t\right\}
$$

The following theorem is thus a direct consequence [6].

Theorem 14 (Double offset theorem). Let $K \subseteq \mathbb{R}^{d}$ be a set with positive $\mu$-reach. Then, for any radius $r$ in $\left(0, \operatorname{reach}_{\mu}(K)\right)$ and every $t<\mu r$ the hypersurface $\partial K^{r, t}$ is $C^{1,1}$-smooth. In addition,

$$
\operatorname{reach}\left(\partial K^{r, t}\right) \geq \min (t, \mu r-t) .
$$

This implies in particular that the smallest of the principal radii of curvature at any point of $\partial K^{r, t}$ is at least $\min (t, \mu r-t)$.

\section{Boundary measures and Federer's curvature mea- sures}

In this section, we introduce Federer's curvature measures, which apply to a large class of compact subsets embedded in the Euclidean space. The main objective here is to prove a stability theorem for these curvature measures which implies that it is possible to estimate them from point cloud data. As a first step, we consider a a simpler notion called boundary measures and introduced in [8] for the purpose of geometric inference.

\section{Boundary measures}

Loosely speaking, the boundary measure associated to a surface encodes the distribution of normals to the surface at a certain scale, and can be used to detect sharp edges, or highly curved areas, where the concentration of normals is much higher.

Definition 15 (Boundary measures). If $K$ is a compact subset and $E$ a domain of $\mathbb{R}^{d}$, the boundary measure $\mu_{K, E}$ is defined as follows: for any subset $B \subseteq \mathbb{R}^{d}, \mu_{K, E}(B)$ is the $d$-volume of the set of points of $E$ whose projection on $K$ is in $B$, i.e.

$$
\mu_{K, E}(B)=\mathcal{H}^{d}\left(p_{K}^{-1}(B \cap K) \cap E\right) .
$$

Here and in the following, $\mathcal{H}^{d}$ denotes the $d$-dimensional Hausdorff measure. By construction, the total mass of this measure is equal to $\mathcal{H}^{d}(E)$. 


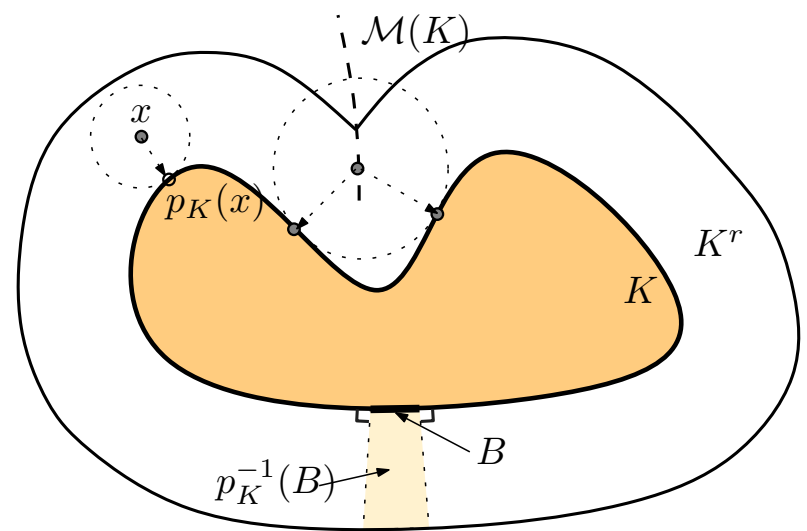

Figure 1: Boundary measure of $K \subset \mathbb{R}^{d}$. The medial axis $\operatorname{Med}(K)$ of $K$ is the dashed line. Remark that the boundary of the offset $\partial K^{r}$ is smooth everywhere but at its point of intersection with the medial axis.

EXAMPLE 16. Let $S$ be a unit-length segment in the plane with endpoints $a$ and $b$. The set $S^{r}$ is the union of a rectangle of dimension $1 \times 2 r$ whose points project on the segment and two half-disks of radius $r$ whose points are projected on $a$ and $b$. It follows that

$$
\mu_{S, S^{r}}=\left.2 r \mathcal{H}^{1}\right|_{S}+\frac{\pi}{2} r^{2} \delta_{a}+\frac{\pi}{2} r^{2} \delta_{b}
$$

EXAmple 17. If $P$ is a convex solid polyhedron of $\mathbb{R}^{3}, F$ its faces, $E$ its edges and $V$ its vertices, then one can see that:

$$
\mu_{P, P^{r}}=\left.\mathcal{H}^{3}\right|_{P}+\left.r \sum_{f \in F} \mathcal{H}^{2}\right|_{f}+\left.r^{2} \sum_{e \in E} H(e) \mathcal{H}^{1}\right|_{e}+r^{3} \sum_{v \in V} K(v) \delta_{v}
$$

where $H(e)$ is the angle between the normals of the faces adjacent to the edge $e$ and $K(v)$ the solid angle formed by the normals of the faces adjacent to $v$. As shown by Steiner and Minkowski, for general convex polyhedra the measure $\mu_{K, K^{r}}$ is a sum of weighted Hausdorff measures supported on the $i$-skeleton of $K$, and whose local density is the local external dihedral angle.

\section{Stability of boundary measures}

In this section, we suppose that $E$ is a fixed open set with rectifiable boundary, and we obtain a quantitative stability theorem for the map $K \mapsto \mu_{K, E}$. 
What we mean by stable is that if the Hausdorff distance between two compact sets $K$ and $P$ is small, then the bounded-Lipschitz distance between the boundary measures $\mu_{K, E}$ and $\mu_{P, E}$ is also small.

Definition 18 (bounded-Lipschitz distance). The bounded-Lipschitz distance between two signed measures $\mu, \nu$ with finite total mass is

$$
\mathrm{d}_{\mathrm{bL}}(\mu, \nu)=\sup _{f}\left|\int f \mathrm{~d} \mu-\int f \mathrm{~d} \nu\right|,
$$

where the supremum is over all 1-Lipschitz function in $\mathbb{R}^{d}$ s.t. $\|f\|_{\infty} \leq 1$.

Theorem 19. If $E$ is a fixed open subset of $\mathbb{R}^{d}$ with rectifiable boundary, for each compact $K \subseteq \mathbb{R}^{d}$, then

$$
\mathrm{d}_{\mathrm{bL}}\left(\mu_{K, E}, \mu_{K^{\prime}, E}\right) \leq \operatorname{const}(E, K, d) \mathrm{d}_{\mathrm{H}}\left(K, K^{\prime}\right)^{1 / 2}
$$

Moreover the constant only depends on the diameter of $K$.

Before proving this theorem, we will first show that the exponent $1 / 2$ in the right-hand side of Equation (17) is optimal.

Lemma 20. There exists a sequence of compact subsets $K_{n}$ of $\mathbb{R}^{d}$ converging to a compact set $K$ and a domain $E$, such that

$$
\mathrm{d}_{\mathrm{bL}}\left(\mu_{K, E}, \mu_{K_{n}, E}\right) \geq \text { const } \cdot \mathrm{d}_{\mathrm{H}}\left(K, K_{n}\right)^{1 / 2}
$$

Proof. Let $K$ be the closed unit disk in the plane, $K_{n}$ be the regular polygon with $n$ edges inscribed in $K$, and let $E$ be the annulus $\mathrm{B}(0,2) \backslash \mathrm{B}(0,1)$. Denote $\ell$ the edgelength of $K_{n}$. Pythagoras theorem can be used to bound the Hausdorff distance between $K$ and $K_{n}$ in term of $\ell_{n}$ : $\mathrm{d}_{\mathrm{H}}\left(K, K_{n}\right) \leq$ const $\cdot \ell_{n}^{2}$. The measure $\mu=\mu_{K, E}$ is proportional to the uniform (lineic) measure on the unit circle. On the other hand, the map $\mathrm{p}_{K_{n}}$ projects a constant fraction of the mass of $E$ onto the vertices $V_{n}$ of $K_{n}$. The cost of spreading out the mass concentrated on these vertices to get a uniform measure on the circle is proportional to the distance between consecutive vertices, so that $\mathrm{d}_{\mathrm{bL}}\left(\mu_{D, E}, \mu_{P_{\ell}, E}\right) \geq$ const $\ell_{n}$.

The next lemma shows that Theorem 19 can be deduced from a $\mathrm{L}^{1}$ stability result for projection functions.

Lemma 21. $\mathrm{d}_{\mathrm{bL}}\left(\mu_{K, E}, \mu_{L, E}\right) \leq\left\|\mathrm{p}_{K}-\mathrm{p}_{L}\right\|_{\mathrm{L}^{1}(E)}$ 
Proof. Consider a 1-Lipschitz function $f$ on $\mathbb{R}^{d}$. Then, by the change of variable formula, and using the Lipschitz property,

$$
\begin{aligned}
\int_{\mathbb{R}^{d}} f(p) \mathrm{d}\left(\mu_{K, E}(p)-\mu_{L, E}(p)\right) & =\int_{E} f\left(\mathrm{p}_{K}(x)\right)-f\left(\mathrm{p}_{L}(x)\right) \mathrm{d} \mathcal{H}^{d}(x) \\
& \leq \int_{E}\left\|\mathrm{p}_{K}(x)-\mathrm{p}_{L}(x)\right\| \mathrm{d} \mathcal{H}^{d}(x)=\left\|p_{K}-\mathrm{p}_{K}\right\|_{L^{1}(E)}
\end{aligned}
$$

Taking the maximum over 1-Lipschitz functions bounded by 1 gives the desired bound.

Proposition 5 allows us to rewrite the projection function $\mathrm{p}_{K}$ as the gradient of a convex function: setting $\psi_{K}(x):=\frac{1}{2}\left(\|x\|^{2}-\mathrm{d}_{K}(x)^{2}\right)$, one has $\mathrm{p}_{K}=\nabla \psi_{K}$. This rewriting recasts a difficult geometric question into a seemingly easier analytical question, namely a $\mathrm{L}^{1}$-stability of gradients of convex functions. This is the object of the next theorem. The proof presented here is different from the original proof in [8] and gives a slightly better constant.

Theorem $22([8])$. Let $\phi, \psi: \mathbb{R}^{d} \rightarrow \mathbb{R}$ be two convex functions and $E$ be a bounded domain with rectifiable boundary. Then,

$$
\|\nabla \phi-\nabla \psi\|_{L^{2}(E)}^{2} \leq 2\|\phi-\psi\|_{L^{\infty}(E)}\left(\|\nabla \phi\|_{L^{\infty}(E)}+\|\nabla \psi\|_{L^{\infty}(E)}\right) \mathcal{H}^{d-1}(\partial E)
$$

Proof. Note that if the inequality (19) holds for smooth convex functions, then it also holds for any convex function. Indeed, it suffices to remark that any convex function $\phi$ can be approximated by a sequence of smooth convex functions $\left(\phi_{n}\right)$ such that $\nabla \phi_{n}$ converges uniformly to $\nabla \phi$ on any compact domain. By another approximation argument, it is possible to replace $E$ by a domain with smooth boundary. From now on, we will assume that $\phi, \psi$ and $\partial E$ are smooth. We use Stokes theorem to get:

$$
\int_{E}\|\nabla \phi-\nabla \psi\|^{2}=\int_{\partial E}(\phi-\psi)\left\langle\nabla \phi-\nabla \psi \mid \mathbf{n}_{E}\right\rangle-\int_{E}(\phi-\psi) \Delta(\phi-\psi)
$$

The first term of this sum is easily bounded, using the fact that an integral is bounded by the maximum of the integrand multiplied by the measure of the domain, and the Cauchy-Schwartz inequality:

$\left|\int_{\partial E}(\phi-\psi)\left\langle\nabla \phi-\nabla \psi \mid \mathbf{n}_{E}\right\rangle\right| \leq\|\phi-\psi\|_{L^{\infty}(E)}\left(\|\nabla \phi\|_{L^{\infty}(E)}+\|\nabla \psi\|_{L^{\infty}(E)}\right) \mathcal{H}^{d-1}(\partial E)$ 
We can bound the second term similarly:

$$
\left|\int_{E}(\phi-\psi) \Delta(\phi-\psi)\right| \leq\|\phi-\psi\|_{L^{\infty}(E)} \int_{E}(|\Delta \phi|+|\Delta \psi|)
$$

We now use the convexity hypothesis, which implies that $\Delta \phi$ is non-negative, which allows us to apply Stokes theorem again:

$$
\int_{E}|\Delta \phi|=\int_{E} \Delta \phi=\int_{\partial E}\left\langle\nabla \phi \mid \mathbf{n}_{E}\right\rangle \leq\|\nabla \phi\|_{L^{\infty}(E)} \mathcal{H}^{d-1}(\partial E)
$$

Combining Equations (22)-(23), we get

$$
\left|\int_{E}(\phi-\psi) \Delta(\phi-\psi)\right| \leq\|\phi-\psi\|_{L^{\infty}(E)}\left(\|\nabla \phi\|_{L^{\infty}(E)}+\|\nabla \psi\|_{L^{\infty}(E)}\right) \mathcal{H}^{d-1}(\partial E)
$$

Finally, Equations (20), (21) and (24) give the desired inequality (19).

Proof of Theorem 19. We introduce the functions $\psi_{K}(x):=\frac{1}{2}\left(\|x\|^{2}-\mathrm{d}_{K}(x)^{2}\right)$, which is convex, and we define $\psi_{K^{\prime}}$ similarly. Now, thanks to Lemma 21 and using the Cauchy-Schwarz inequality, we have

$$
\mathrm{d}_{\mathrm{bL}}\left(\mu_{K, E}, \mu_{K^{\prime}, E}\right) \leq\left\|\mathrm{p}_{K}-\mathrm{p}_{K^{\prime}}\right\|_{\mathrm{L}^{1}(E)} \leq \mathcal{H}^{d}(E)\left\|\mathrm{p}_{K}-\mathrm{p}_{K^{\prime}}\right\|_{\mathrm{L}^{2}(E)} .
$$

We are now ready to apply Theorem 22 . Without loss of generality, we assume that the Hausdorff distance between $K$ and $K^{\prime}$ is bounded by $\operatorname{diam}(K)$ and that $K$ contains the origin. Using $\left\|\mathrm{p}_{K}\right\|_{\infty}=\max _{p \in K}\|p\| \leq \operatorname{diam}(K)$,

$$
\left\|\nabla \psi_{K}\right\|_{L^{\infty}(E)}+\left\|\nabla \psi_{K^{\prime}}\right\|_{L^{\infty}(E)} \leq\left\|\mathrm{p}_{K}\right\|_{\infty}+\left\|\mathrm{p}_{K^{\prime}}\right\|_{\infty} \leq 3 \operatorname{diam}(K) .
$$

Finally, we need to control the quantity $\|\phi-\psi\|_{L^{\infty}(E)}$. For this purpose, we use the relation $\left\|\mathrm{d}_{K}-\mathrm{d}_{K^{\prime}}\right\|_{\infty}=\mathrm{d}_{\mathrm{H}}\left(K, K^{\prime}\right)$ :

$$
\begin{aligned}
\|\phi-\psi\|_{\mathrm{L}^{\infty}(E)} & =\left\|\mathrm{d}_{K}^{2}-\mathrm{d}_{K^{\prime}}^{2}\right\|_{\mathrm{L}^{\infty}(E)} \\
& \leq\left(\left\|\mathrm{d}_{K}\right\|_{\mathrm{L}^{\infty}(E)}+\left\|\mathrm{d}_{K^{\prime}}\right\|_{\mathrm{L}^{\infty}(E)}\right) \mathrm{d}_{\mathrm{H}}\left(K, K^{\prime}\right) \\
& \leq \operatorname{const}(K, E) \mathrm{d}_{\mathrm{H}}\left(K, K^{\prime}\right),
\end{aligned}
$$

where the last inequality follows from the assumption that $\mathrm{d}_{\mathrm{H}}\left(K, K^{\prime}\right) \leq$ $\operatorname{diam}(K)$, which implies that $\left\|\mathrm{d}_{K^{\prime}}\right\|_{L^{\infty}(E)} \leq\left\|\mathrm{d}_{K}\right\|_{L^{\infty}(E)}+\operatorname{diam}(K)$. The stability inequality (17) then follows from (25), Theorem 22 and Equations (26)-(27). 


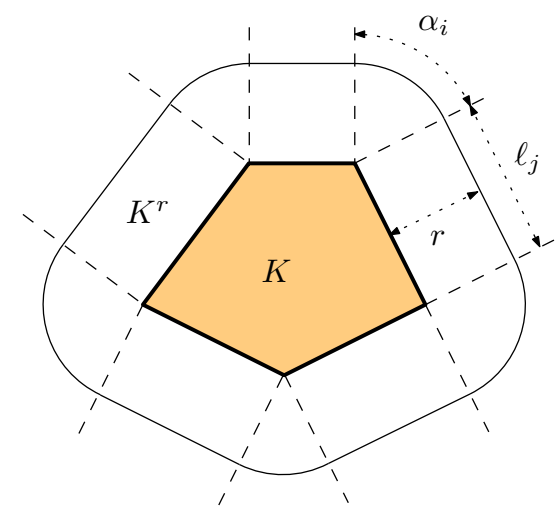

Figure 2: Offset of a polygon in the Euclidean plane

\section{Tube formulas and Federer's curvature measures}

We start with the tube formulas of Steiner, Minkowski and Weyl, before turning to the more precise tube formula of Federer, which is then used to define the curvature measures of a large class of compact sets.

Tube formulas A tube formula for a compact set $K$ in Euclidean space asserts that the Lebesgue volume of the tubular neighborhoods $K^{r}$ is a degree $d$ polynomial in $r$ on a certain interval. The first tube formula is due to Steiner and shows that if $K$ is a convex polygon in the Euclidean plane, the function $r \mapsto \mathcal{H}^{d}\left(K^{r}\right)$ is a polynomial of degree two, and more precisely,

$$
\mathcal{H}^{d}\left(K^{r}\right)=\mathcal{H}^{2}(K)+r \mathcal{H}^{1}(\partial K)+\pi r^{2}
$$

The proof of this fact is (almost) contained in Figure 2: every vertex with exterior angle $\alpha_{i}$ contributes a volume of $\alpha_{i} r^{2}$ to $K^{r}$, while every segment contributes $r \times \ell_{j}$. Summing these up on every segment and vertex yields the $2 D$ Steiner formula. Minkowski proved a similar polynomial behaviour for the volume of the offsets of any compact convex set in $\mathbb{R}^{d}$.

Weyl [25] proved that the polynomial behavior for $r \mapsto \mathcal{H}^{d}\left(K^{r}\right)$ also holds for small values of $r$ when $K$ is a compact smooth submanifold of $\mathbb{R}^{d}$. He also proved that the coefficients of this polynomial can be computed from the second fundamental form of $K$. The following proposition deals with the (simple) case of an hypersurface bounding a compact domain. 
Proposition 23. Let $K$ be a bounded domain of $\mathbb{R}^{d}$ with smooth boundary $M$. Then, for sufficiently small values of $r>0$,

$$
\mathcal{H}^{d}\left(K^{r}\right)=\mathcal{H}^{d}(K)+\sum_{k=1}^{d-1} \operatorname{const}(d, k) r^{k+1} \int_{M}\left[\sum_{i_{1}<\cdots<i_{k}} \kappa_{i_{1}} \ldots \kappa_{i_{k}}(p)\right] \mathrm{d} p
$$

where $\kappa_{1}(p), \ldots, \kappa_{d}(p)$ are the principal curvatures at point $p$ of $\partial K=M$.

Proof. Let $\mathbf{n}$ be an oriented normal field on $M$. The map $\Phi: M \times \mathbb{R} \rightarrow$ $\mathbb{R}^{d},(p, t) \mapsto p+t \mathbf{n}$ is locally injective; by compactness of $M$, it is also injective on $M \times[0, r]$ for $r$ small enough. One has $\mathrm{d}_{(p, t)} \Phi=\mathrm{id}_{\mathrm{T}_{p} M}+t \mathrm{~d}_{p} \mathbf{n}+\mathbf{n}$, i.e. $\left|\operatorname{det}\left(\mathrm{d}_{(p, t)} \Phi\right)\right|=\left|\operatorname{det}\left(\mathrm{id}+t \mathrm{~d}_{p} \mathbf{n}\right)\right|$. For $t=0, \operatorname{det}\left(\mathrm{d}_{(p, t)} \Phi\right)=1>0$ at any point $p \in M$; as a consequence, and by compactness of $M$ again, this determinant remains positive for small enough values of $t$. This allows us to apply a following change-of-variable formula for small valus of $r$ :

$$
\begin{aligned}
\mathcal{H}^{d}\left(K^{r}\right) & =\mathcal{H}^{d}(K)+\int_{K^{r} \backslash K} 1 \mathrm{~d} x \\
& =\mathcal{H}^{d}(K)+\int_{M} \int_{0}^{r} \operatorname{det}\left(\mathrm{id}+t \mathrm{~d}_{p} \mathbf{n}\right) \mathrm{d} t \mathrm{~d} \mathbf{n}
\end{aligned}
$$

The eigenvalues of the map $\mathrm{d}_{p} \mathbf{n}$ are the $d$ principal curvatures of $M$ at $p$, which means:

$$
\operatorname{det}\left(\mathrm{id}+t \mathrm{~d}_{p} \mathbf{n}\right)=\prod_{i=1}^{d-1}\left(1+t \kappa_{i}(p)\right)=\sum_{k=1}^{d} t^{k}\left[\sum_{i_{1}<\cdots<i_{k}} \kappa_{i_{1}}(p) \ldots \kappa_{i_{k}}(p)\right]
$$

We conclude the proof by putting Equation (31) in Equation (30).

Federer's curvature measures The contribution of Federer to the theory of tube formulas is twofolds. First, Federer defines the class of compact sets with positive reach, which includes both compact convex sets and compact smooth submanifolds of $\mathbb{R}^{d}$. The reach of a compact subset of $\mathbb{R}^{d}$ is an interesting quantity, because it gives a lower bound on the largest $R$ such that the map $r \mapsto \mathcal{H}^{d}\left(K^{r}\right)$ is a polynomial of degree $d$ on $[0, R]$. Second, Federer associates to any set with positive reach a family of $d+1$ curvature measures, which allow one to recover local curvature information. The construction of these curvature measures rely on a local version of the tube formula [12].

As mentioned in Section 1.1, sets with positive reach generalize both convex sets and compact smooth submanifolds. In order to introduce the 
curvature measures of a set $K$ with positive reach, Federer first proves that for any positive $r$ in $(0, \operatorname{reach}(K)]$, the boundary of the offset $\partial K^{r}$ is a hypersurface of class $\mathcal{C}^{1,1}$, i.e. $\partial K^{r}$ is a $\mathcal{C}^{1}$ hypersurface with a Lipschitz normal field. Federer's then extends the tube formula presented in Proposition 23 to this less smooth setting. Finally, the existence of curvature measures for $K$, as well as the polynomial behaviour for the volume of the offsets is obtained by approximation, by letting $r$ go to zero. We will not prove these facts, but only quote Federer's existence result. As usual, $\omega_{k}$ denotes the volume of the $k$-dimensional sphere in $\mathbb{R}^{k+1}$.

Theorem 24 (Federer). For any compact set $K \subseteq \mathbb{R}^{d}$ with positive reach, there exists $d+1$ signed measures $\Phi_{K, 0}, \ldots, \Phi_{K, d}^{d}$ such that for $r \leq \operatorname{reach}(K)$,

$$
\mu_{K, K^{r}}=\sum_{i=0}^{d} \omega_{d-i} \Phi_{K, i} r^{i} .
$$

Definition 25. The measures $\Phi_{K, 0}, \ldots, \Phi_{K, d}$ introduced in Theorem 24 are called Federer's curvature measures of $K$.

\section{Stability of Federer's curvature measures}

The purpose of this section is to show that Federer's curvature measures of a compact set with positive reach can be estimated from a Hausdorff approximation of this set, without any regularity hypothesis on the approximating set. In order to do so, we explain how to associate an ersatz of Federer's curvature measures to any compact set, which coincide with the original curvature measures when the set has a sufficiently large reach.

Definition 26 (Approximate curvature measures). Let $R>0$ and $r$ be a family of numbers $\left(r_{i}\right)_{0 \leq i \leq d}$ such that $0<r_{0}<\ldots<r_{d}$. Given any compact set $K$ in $\mathbb{R}^{d}$ and any Borel set $B$, we define the approximate curvatures $\left(\Phi_{K, i}^{(r)}(B)\right)_{0 \leq i \leq d}$ as the set of coefficients which satisfy the interpolation equations:

$$
\forall 0 \leq i \leq d-1, \mu_{K, K^{r}}(B)=\sum_{j=0}^{d} \omega_{d-j} \Phi_{K, j}^{(r)}(B) r_{i}^{j}
$$

Since the numbers $\left(r_{i}\right)$ are distinct, this define the approximate curvature measures uniquely. More precisely, by Lagrange interpolation, there exist real coefficients $\left(L_{i j}\right)$ such that

$$
\forall 0 \leq i \leq d-1, \Phi_{K, j}^{(r)}=\sum_{0 \leq i \leq d} L_{i j} \mu_{K, K^{r_{i}}} .
$$


This shows that the approximate curvature measure $\Phi_{K, j}^{(r)}$ is a signed measure. Moreover, one recovers Federer's curvature measures when the reach of $K$ is sufficiently large. More precisely, if $r_{d} \leq \operatorname{reach}(K)$, then $\Phi_{K, j}^{(r)}=\Phi_{K, j}$.

We are now able to state the following stability theorem from [8].

Theorem 27. Given any compact set $K \subseteq \mathbb{R}^{d}$ and $(r)$ as in Definition 26, there exist a constant $C=\operatorname{const}(K, r, d)$ such that for any compact set $K^{\prime}$ sufficiently close to $K$,

$$
\mathrm{d}_{\mathrm{bL}}\left(\Phi_{K, j}^{(r)}, \Phi_{K^{\prime}, j}^{(r)}\right) \leq C \mathrm{~d}_{\mathrm{H}}\left(K, K^{\prime}\right)^{1 / 2}
$$

Moreover, if $r_{d} \leq \operatorname{reach}(K)$ then one can estimate Federer's curvature measures of $K$ from $K^{\prime}$ :

$$
\mathrm{d}_{\mathrm{bL}}\left(\Phi_{K, j}^{(r)}, \Phi_{K^{\prime}, j}\right) \leq C \mathrm{~d}_{\mathrm{H}}\left(K, K^{\prime}\right)^{1 / 2} .
$$

We will see first how Theorem 27 can be deduced from the following stability result for boundary measures.

Theorem 28. Given any compact set $K \subseteq \mathbb{R}^{d}$ and $r$ a positive number, there exist a constant $C=\operatorname{const}(K, r, d)$ such that for any compact set $K^{\prime}$ sufficiently close to $K$,

$$
\mathrm{d}_{\mathrm{bL}}\left(\mu_{K, K^{r}}, \mu_{K^{\prime}, K^{\prime r}}\right) \leq C \mathrm{~d}_{\mathrm{H}}\left(K, K^{\prime}\right)^{1 / 2} .
$$

Proof of Theorem 28. Let $E$ be the symmetric difference between $K^{r}$ and $K^{\prime r}$. Then, by the triangle inequality for the bounded-Lipschitz distance,

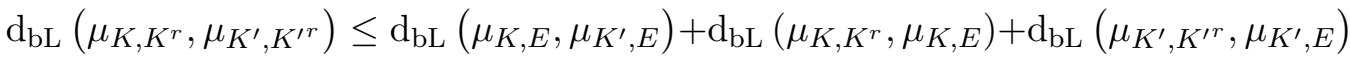

Theorem 19 implies that the first term of the right-hand side is of order $\mathrm{O}\left(\mathrm{d}_{\mathrm{H}}^{1 / 2}\left(K, K^{\prime}\right)\right)$. We only need to deal with the two last terms. Given any 1Lipschitz function $f$ with $\|f\|_{\infty} \leq 1$, the change-of-variable formula implies

$$
\begin{aligned}
\int_{\mathbb{R}^{d}} f(x) \mathrm{d}\left(\mu_{K, K^{r}}-\mu_{K, E}\right) & \leq \int_{K^{r}} f\left(\mathrm{p}_{K}(x)\right) \mathrm{d} \mathcal{H}^{d}(x)-\int_{E} f\left(\mathrm{p}_{K}(x)\right) \mathrm{d} \mathcal{H}^{d}(x) \\
& \leq \mathcal{H}^{d}\left(K^{r} \backslash E\right)
\end{aligned}
$$

Taking the maximum over such functions gives a bound on the boundedLipschitz distance between these measures. Overall, we have

$$
\mathrm{d}_{\mathrm{bL}}\left(\mu_{K, K^{r}}, \mu_{K^{\prime}, K^{\prime r}}\right) \leq C \mathrm{~d}_{\mathrm{H}}^{1 / 2}\left(K, K^{\prime}\right)+\mathcal{H}^{d}\left(K^{r} \backslash E\right)+\mathcal{H}^{d}\left(K^{\prime r} \backslash E\right) .
$$

Defining $\varepsilon=\mathrm{d}_{\mathrm{H}}\left(K, K^{\prime}\right)$, one has the inclusion $K^{r} \backslash E \subseteq K^{r} \backslash K^{r-\varepsilon}$. This term can be shown to be of order $\mathrm{O}\left(\mathrm{d}_{\mathrm{H}}\left(K, K^{\prime}\right)\right)$ using Proposition 4.2 from [8] and the coarea formula. 


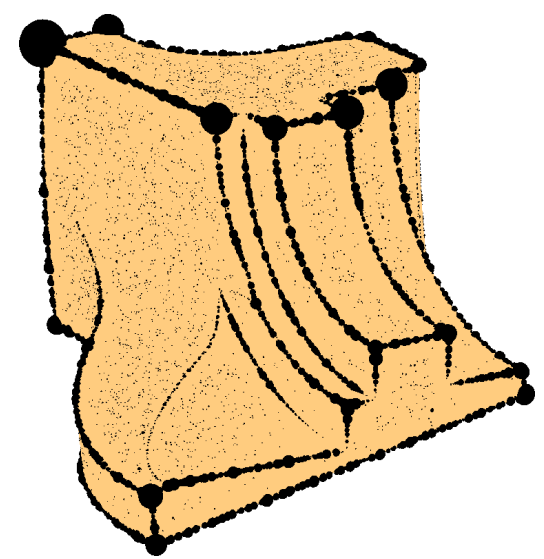

(a) Fandisk

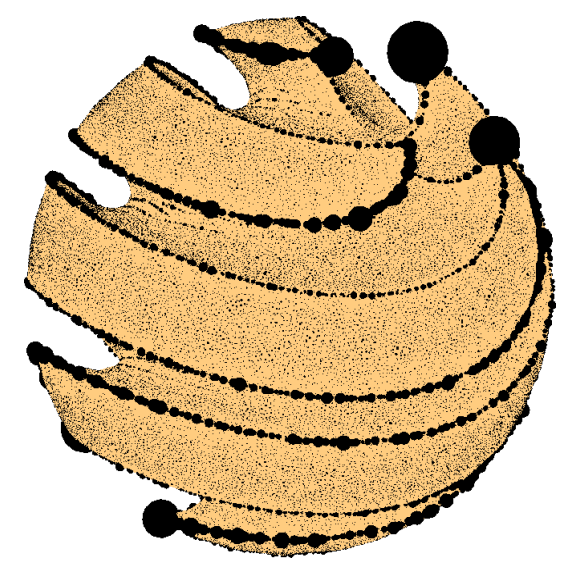

(b) Sharp sphere

Figure 3: Convolved boundary measures of $100 \mathrm{k}$ point clouds sampled from point clouds sampled from the fandisk and sharp sphere models and rescaled so as to have unit diameter. The offset radius is set to $r=0.05$ and $r=0.1$ respectively and the convolution radius is given by $\varepsilon=0.02$ and $\varepsilon=0.03$.

Proof of Theorem 27. Thanks to Equation (34), one has for any 1-Lipschitz function $f$ such that $\|f\|_{\infty} \leq 1$,

$$
\begin{aligned}
\left|\int_{\mathbb{R}^{d}} f \mathrm{~d}\left(\Phi_{K, j}^{(r)}-\Phi_{K^{\prime}, j}^{(r)}\right)\right| & \leq \sum_{0 \leq i \leq d}\left|L_{i j}\right|\left|\int_{\mathbb{R}^{d}} f \mathrm{~d}\left(\mu_{K, K^{r_{i}}}-\mu_{K, K^{r_{i}}}\right)\right| \\
& \leq \sum_{0 \leq i \leq d}\left|L_{i j}\right| \mathrm{d}_{\mathrm{bL}}\left(\mu_{K, K^{r_{i}}}, \mu_{K, K^{r_{i}}}\right) \\
& \leq \operatorname{const}(K,(r), d) \mathrm{d}_{\mathrm{H}}\left(K, K^{\prime}\right)^{1 / 2},
\end{aligned}
$$

where the second inequality follows from the definition of the boundedLipschitz distance, and the third inequality comes from Theorem 28. To conclude, it suffices to apply the definition of the bounded-Lipschitz distance once again.

\section{Computation of boundary measures and visualization}

We explain briefly how to compute the boundary measure of a point cloud, that is a finite subset $P$ of $\mathbb{R}^{d}$. The Federer's curvature measures can be recovered from the boundary measures through polynomial fitting. The 
computation relies on the Voronoi diagram of $P$, which is a practical way of encoding the distance function to $P$ in low dimension.

Definition 29 (Voronoi diagram). Let $P$ be a point cloud of $\mathbb{R}^{d}$. The Voronoi diagram of $P$ is a decomposition of the space into convex polyhedra called Voronoi cells. The Voronoi cell of the point $p$ in $P$ is defined by:

$$
\operatorname{Vor}_{P}(p)=\left\{x \in \mathbb{R}^{d} ; \forall q \in P,\|x-p\| \leq\|x-q\|\right\} .
$$

Lemma 30. Let $P$ be a point cloud of $\mathbb{R}^{d}$. The boundary measure of $P$ with respect to a domain $E$ is given by

$$
\mu_{P, E}=\sum_{p \in P} \mathcal{H}^{d}\left(\operatorname{Vor}_{P}(p) \cap E\right) \delta_{p}
$$

In addition, when $E=P^{r}$ is an offset of $P$, one has

$$
\mu_{P, P^{r}}=\sum_{p \in P} \mathcal{H}^{d}\left(\operatorname{Vor}_{P}(p) \cap \mathrm{B}(p, r)\right) .
$$

Proof. Equation (36) follows from the fact that a point $x$ belongs to the Voronoi cell of $p$ in $P$ if and only if $\mathrm{d}_{P}(x)=p$ and iff $\mathrm{p}_{P}(x)=p$. To prove the second equation, we only need to remark that

$$
\begin{aligned}
\operatorname{Vor}_{P}(p) \cap P^{r} & =\left\{x \in \mathbb{R}^{d} ; \mathrm{d}_{P}(x)=\|x-p\| \text { and } \mathrm{d}_{P}(x) \leq r\right\} \\
& =\left\{x \in \mathbb{R}^{d} ; \mathrm{d}_{P}(x)=\|x-p\| \text { and }\|x-p\| \leq r\right\} \\
& =\operatorname{Vor}_{P}(p) \cap \mathrm{B}(p, r)
\end{aligned}
$$

Thanks to this lemma, computing boundary measures amounts to evaluating the volume of intersections of Voronoi cells with balls. This leads to a practical algorithm in dimension 2 and 3 [20]. This approach becomes too costly in higher dimensions due to the exponential complexity of the Voronoi diagram, but it is possible to resort to a Monte-Carlo method [8].

Visualization It is not trivial how to visualize a finitely supported measure $\mu_{P, P^{r}}$ on $\mathbb{R}^{d}$, even when the ambient dimension is $d=2,3$. The obvious idea is to display a ball whose volume is proportional to the mass of the Dirac at each point in the support of the measure. This is however not satisfactory as two measures which are close for the bounded-Lipschitz distance could lead to very different visualizations. Indeed, consider $\mu=\delta_{x}$ and $\mu_{\eta}=\left(\delta_{x}+\delta_{y}\right) / 2$ where $\|x-y\| \leq \eta: \mu$ would be displayed as a single ball $\mathrm{B}(x, r)$ while the 


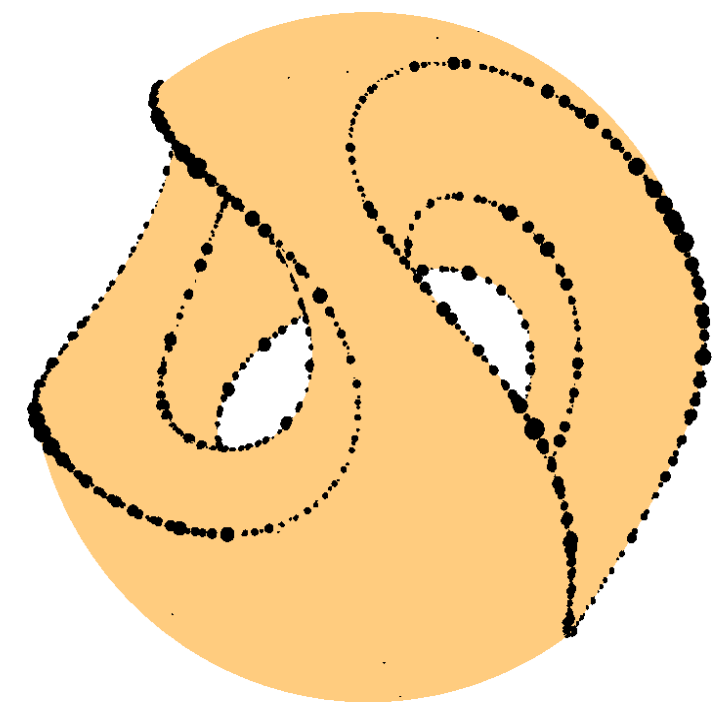

Figure 4: Feature points extracted from a point cloud sampling of a CSG model by thresholding low values of the convolved boundary measure.

nearby measure $\mu_{\eta}$ would be displayed as two overlapping balls of smaller radius $\mathrm{B}(x, \alpha r) \cup \mathrm{B}(x, \alpha r)$ with $\alpha=2^{-1 / d}$.

In order to construct a visualization which is stable with respect to the bounded-Lipschitz distance, we therefore convolve each boundary measure with a fixed bounded-Lipschitz function $\chi$.

Definition 31 (Convolution). The convolution of a finite measure $\mu$ on $\mathbb{R}^{d}$ with a bounded measurable function $\chi$ is the function $\mu * \chi: \mathbb{R}^{d} \rightarrow \mathbb{R}$ defined by

$$
[\mu * \chi](x):=\int_{\mathbb{R}^{d}} \chi(y-x) \mathrm{d} \mu(y)
$$

The convolved measure $\mu * \chi$ is stable with respect to the boundedLipschitz distance, by its very definition. More precisely,

Lemma 32. Let $\chi$ be a function on $\mathbb{R}^{d}$ such that $\operatorname{Lip} \chi \leq 1$ and $\|\chi\|_{\infty} \leq 1$. Then for finite measures $\mu, \nu$,

$$
\|\mu * \chi-\nu * \chi\|_{\infty} \leq \mathrm{d}_{\mathrm{bL}}(\mu, \nu)
$$

In practice, we choose the convolution kernel to be the "hat function" $\chi_{\varepsilon}(y)=\max (\varepsilon-\|x-y\|, 0)$, and we display at each point in $P$ a ball whose volume is proportional to the value of the function $\mu_{P, P^{r}} * \chi_{\varepsilon}$. Figure 3 shows 
the convolved boundary measures of point clouds sampling piecewise-smooth surfaces in $\mathbb{R}^{3}$.

Sharp features extraction Extracting the set of sharp features of a compact sets known through a finite point cloud sampling is of interest in many geometry processing applications. Figure 3 suggests that the sharp corners carry more mass than the points on the sharp edges, which again carry more mass than the smooth points. This observation can be turned into more quantitative estimations, see e.g. [19, Chapter 3]. In Figure 4 we display points whose boundary measures carries more mass than some threshold, or more precisely (for the same reasons as in the previous paragraph), where the values of the function $\mu_{K, K^{r}} * \chi_{\varepsilon}$ are higher than some threshold.

\section{Voronoi covariance measures and local Minkowski tensors}

In some applications, such as feature-aware surface reconstruction, scalar quantities such as those encoded in Federer's curvature measures are not sufficient, and one also wishes to recover directional information such as principal curvature directions or the direction of a sharp edge in a piecewise smooth surface.

\section{Covariance matrices of Voronoi cells}

Voronoi-based normal estimation $[2,1]$ rely on the intuition that for a noiseless sampling of a smooth surface the Voronoi cells are elongated in the direction of the normal to the surface. For instance, in Figure 5, the Voronoi cell of the red point on upper face is an elongated cylinder, and it is possible to estimate the normal to that face by analyzing the shape of this Voronoi cell. A practical tool for estimating the direction in which a domain is elongated is the notion of covariance matrix.

DEFINITION 33 (Covariance matrix). The covariance matrix of a bounded domain $V \subseteq \mathbb{R}^{d}$ is the symmetric matrix, or tensor, defined by:

$$
\operatorname{cov}(V, p)=\int_{V}(x-p) \otimes(x-p) \mathrm{d} x
$$

where $v \otimes w$ denotes the $n \times n$ matrix defined by $[v \otimes w]_{i, j}=w_{i} v_{j}$. 


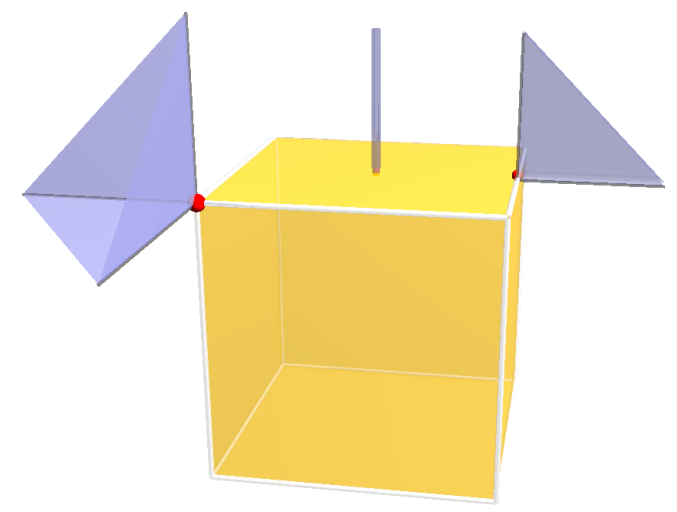

Figure 5: The Voronoi cell of a point $x$ on a cube is pencil, triangle or cone-shaped depending on the dimension of the normal cone.

The covariance matrix of a domain is also known as the inertia matrix in solid mechanics and its eigenvectors capture the principal axes of the domain $V$ with respect to the base point $p$. In the case of a noiseless sampling of a smooth surface, one can approximate the normal direction at each sample by the eigenvector corresponding to the largest eigenvalue of the covariance matrix of the corresponding Voronoi cell intersected with a bounding box of the point set [1]. Under strong noise, individual Voronoi cells can become ill shaped, but it is possible to average the covariance matrices of nearby Voronoi cells to recover the correct normal directions.

Note, however, that the shapes of Voronoi cells provide more information than just the normal direction. When the underlying surface is not smooth, some of its points will have normal cones rather than single normal directions. Nevertheless, even in this case, the shapes of Voronoi cells accurately reflect the shapes of the underlying normal cones. Some geometric properties of these normal cones can then be estimated using the covariance matrices of the Voronoi cells.

\section{Voronoi covariance measure}

It is possible to mimick the definition of the boundary measure to construct a tensor-valued measure which summarizes and extends the covariance matrices of Voronoi cells.

Definition 34. The Voronoi covariance measure (also called VCM) of a compact subset $K$ of $\mathbb{R}^{d}$ with respect to a bounded domain $E$ a tensorvalued measure denoted by $\mathcal{V}_{K, R}$. This measure maps every (Borel) subset 
$B$ of $\mathbb{R}^{d}$ to the symmetric matrix defined by:

$$
\mathcal{V}_{K, E}(B):=\int_{E \cap \mathrm{p}_{K}^{-1}(B \cap K)}\left(x-\mathrm{p}_{K}(x)\right) \otimes\left(x-\mathrm{p}_{K}(x)\right) \mathrm{d} x
$$

Example 35. The Voronoi covariance measure of a point cloud $P \subseteq \mathbb{R}^{d}$ summarizes the covariance matrices of Voronoi cells. More precisely, the Voronoi covariance measure of $P$ with respect to a bounded domain $E$ is a sum of Dirac masses. The weight in front of each Dirac is the covariance matrix of the corresponding Voronoi cell. More precisely,

$$
\mathcal{V}_{K, E}=\sum_{p \in P} \operatorname{cov}\left(\operatorname{Vor}_{P}(p), p\right) \delta_{p}
$$

These quantities can be computed efficiently, provided that one is able to compute (or approximate) the intersection between the Voronoi cell and $E$.

Definition 36. The bounded-Lipschitz distance between two measures $\mu, \nu$ taking values in a normed vector space $(X,\|\cdot\|)$ is defined by

$$
\mathrm{d}_{\mathrm{bL}}(\mu, \nu)=\sup _{f}\left\|\int f \mathrm{~d} \mu-\int f \mathrm{~d} \nu\right\| .
$$

For tensor-valued measure, the vector space is the space of symmetric matrices and $\|$.$\| is the operator norm.$

The Hausdorff stability result for boundary measures (Theorems 19 and 28) can be generalized to Voronoi covariance measures. The VCM can be used for the estimation of the location and direction of sharp features, leading to a practical and efficient algorithm [20].

Theorem 37. Given any compact set $K \subseteq \mathbb{R}^{d}$ and $r$ a positive number, there exist a constant $C=\operatorname{const}(K, r, d)$ such that for any compact set $K^{\prime}$ sufficiently close to $K$,

$$
\mathrm{d}_{\mathrm{bL}}\left(\mathcal{V}_{K, K^{r}}, \mathcal{V}_{K^{\prime}, K^{\prime r}}\right) \leq C \mathrm{~d}_{\mathrm{H}}\left(K, K^{\prime}\right)^{1 / 2}
$$

Extensions The Voronoi covariance measure is closely related to the notion of local Minkowski tensor, which was recently introduced by Hug and Schneider [17]. Theorems 37 and 27 have been extended to this setting by Hug, Kiderlen and Svane [16]. A robust variant of the Voronoi covariance measure is introduced and studied in the $\mathrm{PhD}$ thesis of Cuel [10]. 


\section{Stability of anisotropic curvature measures}

In this last section, we consider the question of approximating anisotropic curvature measures of a compact set from a Hausdorff-approximation. Here, we assume that the unknown compact set $K$ has positive $\mu$-reach: this include smooth manifolds, convex domains and triangulations (see §1.2). We show that it is possible to approximate the anisotropic curvature measures of $K^{r}$ from those of $K^{\prime r}$, where $K^{\prime}$ is a Hausdorff approximation of $K$ and where $r$ lies in some range. In practical applications, the second set $K^{\prime}$ is a point cloud and its offset $K^{\prime r}$ is a finite union of balls, whose anisotropic curvature measures can be computed.

\section{Anisotropic curvature measures of sets with positive reach}

Let $V$ be a compact set with positive reach and let $t$ in $(0, \operatorname{reach}(V))$. Since the hyper-surface $\partial V^{t}$ is of class $C^{1,1}$, the second fundamental form and the principal curvatures of $\partial V^{t}$ are defined almost everywhere. One can therefore define the anisotropic curvature measure introduced in [9].

Definition 38. Let $V$ be a compact set with positive reach. The anisotropic curvature measure of $V$ associates to any Borel set $B$ the matrix

$$
\mathrm{H}_{V}(B)=\lim _{t \rightarrow 0} \int_{\partial V^{t} \cap p_{V}^{-1}(B)} H_{\partial V^{t}}(p) d p
$$

where $H_{\partial V^{t}}$ is a matrix-valued function defined on $\mathbb{R}^{d}$ that coincides with the second fundamental form of $\partial V^{t}$ on the tangent space, and vanishes on the orthogonal component.

Remark that this definition is coherent with the Federer curvature measures. Indeed, the $k^{\text {th }}$ Federer curvature measures satisfies for every Borel subset $B$ of $\mathbb{R}^{d}$ :

$$
\Phi_{V, k}(B)=\lim _{t \rightarrow 0} \int_{\partial V^{t} \cap \mathrm{p}_{V}^{-1}(B)} s^{k}(p) d p,
$$

where $\mathrm{p}_{V}$ is the projection onto $V$ and $s^{k}$ is the k-th elementary symmetric polynomial of the principal curvatures $\lambda_{1}, \ldots, \lambda_{d-1}$ of $\partial V^{t}$.

Now, let $K$ be a compact set whose $\mu$-reach is greater than $r>0$. Then $V=\overline{\mathbb{R}^{d} \backslash K^{r}}$ has a reach greater than $\mu r$. It is then possible to define the curvature measures of $K^{r}[23,24]$ by:

$$
\Phi_{K^{r}}^{k}(B)=(-1)^{k} \Phi_{V}^{k}(B) \quad \text { and } \quad \mathrm{H}_{K^{r}}(B)=-\mathrm{H}_{V}(B) .
$$




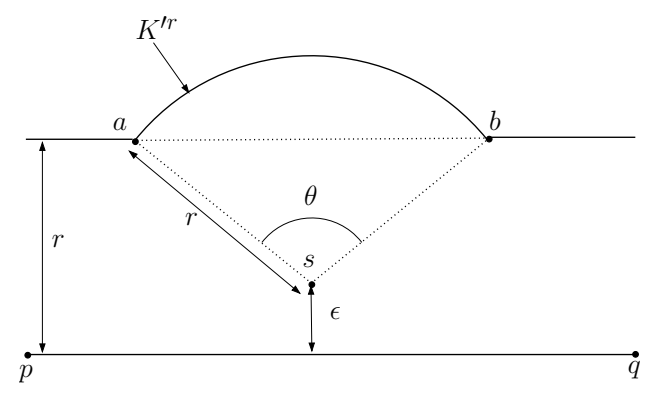

Figure 6: Tightness of the bound of Theorem 39. We consider compact sets $K=[p, q]$ and $K^{\prime}=[p, q] \cup\{s\}$, where $s$ is at a distance $\epsilon$ from $K$. We have $d_{H}\left(K, K^{\prime}\right)=\epsilon$ and the total curvature $\theta$ of $K_{r}^{\prime}$ between $a$ and $b$ satisfies $\theta=$ $2 \arccos \left(\frac{r-\epsilon}{r}\right)=O(\sqrt{\epsilon})$.

\section{Stability of the curvature measures of the offsets}

The following theorem states that if a compact set $K$ is close in the Hausdorff sense to a compact set $K^{\prime}$ with positive $\mu$-reach, then the Federer curvature measures and anisotropic curvature measure of the offsets $K^{r}$ and $K^{\prime r}$ are close for the bounded-Lipschitz distance. This result is similar but not equivalent to Theorem 27. The result in Theorem 27 is limited to the Federer curvature measures but it derives from Theorem 28 , which holds without any assumptions on the underlying compact set, whereas Theorem 39 requires to assume a lower bound on the $\mu$-reach. We recall that the bounded-lipschitz distance is given in Definition 36.

Theorem 39. Let $r>0, K$ and $K^{\prime}$ be two compact subsets of $\mathbb{R}^{d}$ such that $\operatorname{reach}_{\mu}\left(K^{\prime}\right)>r$. We suppose that the Hausdorff distance $\varepsilon=d_{H}\left(K, K^{\prime}\right)$ between $K$ and $K^{\prime}$ is such that $\varepsilon<\frac{\mu^{2}}{60+9 \mu^{2}} r$. Then one has

$$
\mathrm{d}_{\mathrm{bL}}\left(\Phi_{K^{r}, k}, \Phi_{K^{\prime r}, k}\right) \leq \operatorname{const}(r, \mu, d, K) \sqrt{\epsilon}
$$

and

$$
\mathrm{d}_{\mathrm{bL}}\left(\mathrm{H}_{K^{r}}, \mathrm{H}_{K^{\prime r}}\right) \leq \operatorname{const}(r, \mu, d, K) \sqrt{\epsilon},
$$

where const $(r, \mu, d, K)$ depends on $r, \mu, d$ and the diameter of $K$.

This theorem implies that one can estimate locally the curvature measures of a compact set $K^{\prime}$ with positive $\mu$-reach from an Hausdorff approximation. If $f: \mathbb{R}^{d} \rightarrow \mathbb{R}$ is measurable function, the previous theorem implies that

$$
\left|\int f \mathrm{~d} \Phi_{K^{r}, k}-\int f \mathrm{~d} \Phi_{K^{\prime r}, k}\right| \leq \operatorname{const}(r, \mu, d, K)\|f\|_{\mathrm{BL}} \sqrt{\epsilon}
$$


where $\|f\|_{\mathrm{BL}}=\operatorname{Lip}(f)+\|f\|_{\infty} \|$ is the bounded-lipschitz norm of $f$. The similar inequality holds for the anisotropic curvature measure. In practice, we take the hat function $f(x)=\max (1-\|x-c\| / r, 0)$ equal to 1 at a point $c \in \partial K^{\prime r}$. Then we can retrieve local information about the curvature of $K^{\prime r}$ from the curvature of $K^{r}$ in the neighborhood of $c$.

As illustrated in Figure 6, the upper bound of Theorem 39 is tight. However the constant const $(r, \mu, d, K)$ in Equation (43) can be localized. It does not have to depend on the whole compact set $K$, but can only depend on the diameter of the support spt $f=\left\{x \in \mathbb{R}^{d}, f(x) \neq 0\right\}$ of $f$. See [7] for more details.

\section{Computation of the curvature measures of $3 D$ point clouds}

When the compact set $K$ is a finite point set in $\mathbb{R}^{3}$ it is possible to provide explicit formula for the curvature measures. The boundary of $K^{r}$ is a spherical polyhedron: its faces are spherical polygons; its edges are circle arcs contained in the intersection of pairs of spheres of radius $r$ with centers in $K$; its vertices belong to the intersection of three spheres of radius $r$ with centers in $K$. One has explicit integral formula for the curvature measures for each vertex/edge/face of the spherical polygon [7]. Moreover, the combinatorial structure of $\partial K^{r}$ is in one-to-one correspondence with the boundary of the $\alpha$-shape of $K$ [11].

In Figures 7 and 8 below, the curvatures are represented on the boundary of the $\alpha$-shape (for $\alpha=r$ ) of the point clouds where each triangle is colored according to the curvature value of its corresponding vertex in $\partial K^{r}$ and to the colorbar on the right of Figure 7. Note that the color values are different for the different examples (since the extrema values are different). This algorithm can be easily adapted to calculate the anisotropic curvature measures for a finite set of points. In particular, this allows to estimate the principal curvatures and principal directions from a point set.

\section{Sketch of proof of Theorem 39}

We first need to introduce the notion of normal cycle for sets with positive reach. Let $V$ be a set with positive reach. We define the set:

$$
S(V)=\left\{(p, n) \in \mathbb{R}^{d} \times \mathbb{S}^{d-1}, p \in \partial V \text { and } n \in \operatorname{Nor}_{V}(p)\right\},
$$

where $\operatorname{Nor}_{V}(p):=\left\{v \in \mathbb{R}^{d} ; \exists t>0, \mathrm{p}_{V}(p+t v)=p\right\}$ is the normal cone of $V$ at $p$. One can show that $S(V)$ is a Lipschitz (d-1)-manifold. The normal 

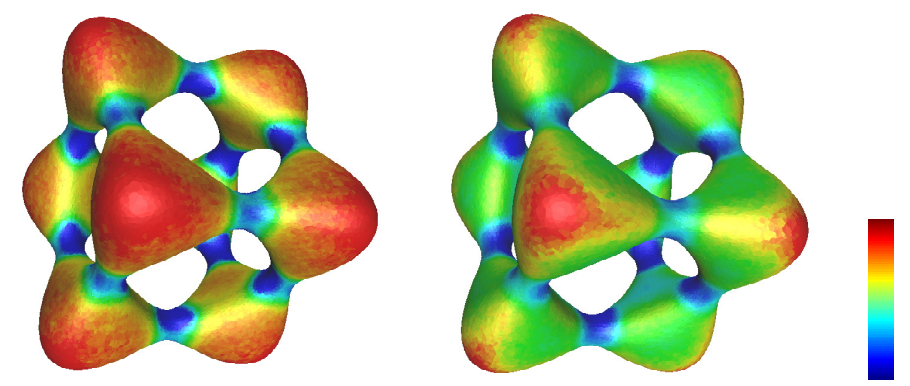

Figure 7: The Gauss (left) and mean (right) curvatures computed on the offset of a point set sampled around a smooth surface. The colors are related to the values of the curvature according to the colorbar on the right, the blue color corresponding to the lowest values.
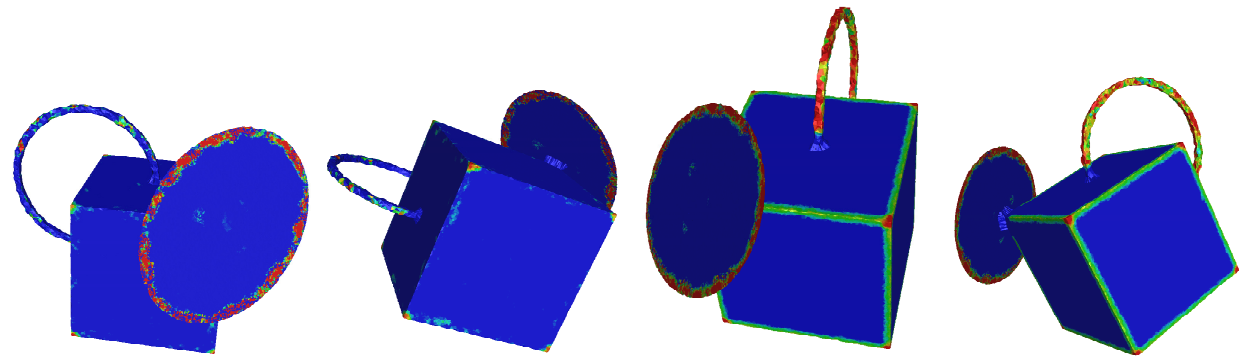

Figure 8: The Gauss (first and second image) and mean (third and fourth image) curvatures computed on the offset of a point cloud sampled around a non-manifold set union of a cube with a disc and a circle. As expected, the vertices and the boundary of the disc have a large Gaussian curvature. 
cycle $N(V)$ of $V$ is then by definition the (d-1)-current on $\mathbb{R}^{d} \times \mathbb{R}^{d}$ defined for every (d-1)-differential form $\omega$ by:

$$
N(V)(\omega)=\int_{S(V)} \omega
$$

The normal cycle contains in fact all the curvature information and allows to define the curvature measures [9]. For every $k$, the curvature measure $\Phi_{V, k}(B)$ is given by $N(V)\left(\bar{f} \omega_{k}\right)$ where $\bar{f}(p, n)=\mathbf{1}_{B}(p)$ and $\omega_{k}$ is a $d-1$ differential form on $\mathbb{R}^{d} \times \mathbb{R}^{d}$ that does not depend on $V$. Similarly, the anisotropic curvature curvature measure $\bar{H}_{V}(X, Y)$ applied in the directions $X$ and $Y$ is given by $N(V)\left(\bar{f} \omega_{H}^{X, Y}\right)$, where $\omega_{H}^{X, Y}$ is a $d$-1-differential form that depends on $X$ and $Y$.

The proof can now be divided into three steps: in the first step, we show that the problem can be carried onto the double offsets (that are smooth); in a second step, we compare the normal cycles of the double offsets; in the last step, we combine Step 1 and Step 2 to show that the curvature measures of the two offsets are close. Let $K$ and $K^{\prime}$ be two compact sets with positive $\mu$-reach that satisfy all the assumptions of Theorem 39 .

\section{Step 1: Carrying the problem into the double offsets}

First note that $\overline{\mathbb{R}^{d} \backslash K^{r}}$ and $\overline{\mathbb{R}^{d} \backslash K^{\prime r}}$ have positive reach. We introduce the map:

$$
\begin{aligned}
F_{-t}: \mathbb{R}^{d} \times \mathbb{R}^{d} & \rightarrow \mathbb{R}^{d} \times \mathbb{R}^{d} \\
(p, n) & \mapsto(p-t n, n)
\end{aligned}
$$

If $V$ is any compact set with positive reach, the map $F_{-t}$ induces naturally a one-to-one correspondence between the support of the normal cycle of the offset $V^{t}$ and the support of the normal cycle of $V$. In particular, this map allows to send simultaneously the normal cycles of $K^{r, t}$ and $K^{\prime r, t}$ to respectively the normal cycles of $K^{r}$ and $K^{\prime r}$. More precisely, one has:

$$
N\left(\overline{\mathbb{R}^{d} \backslash K^{r}}\right)-N\left(\overline{\mathbb{R}^{d} \backslash K^{\prime r}}\right)=F_{-t \sharp}\left(N\left(K^{r, t}\right)-N\left(K^{r, t}\right)\right),
$$

where $F_{-t \sharp}$ denotes the push-forward for currents. Therefore, in order to compare the normal cycles of $\overline{\mathbb{R}^{d} \backslash K^{r}}$ and $\overline{\mathbb{R}^{d} \backslash K^{\prime r}}$, it is sufficient to compare the normal cycles of the double offsets $K^{r, t}$ and $K^{\prime r, t}$.

\section{Step 2: Comparison of the normal cycles of the double offsets}

In order to compare the normal cycles of $K^{r, t}$ and $K^{\prime r, t}$, we first need to compare their (geometrical) supports in $\mathbb{R}^{d} \times \mathbb{R}^{d}$. One first shows that the Hausdorff distance between $\partial K^{r, t}$ and $\partial K^{\prime r, t}$ is less than $\epsilon / \mu$. Using a result of [4] one also shows that the difference between the normals of $\partial K^{r, t}$ 
and $\partial K^{\prime r, t}$ is bounded by $30 \sqrt{\epsilon /(\mu t)}$. Hence the (geometrical) supports of $N\left(K^{r, t}\right)$ and $N\left(K^{r, t}\right)$ are close to each other. Let us take $t=\mu r / 2$. Since the reach of $\partial K^{r, t}$ is larger than $t$, the projection map $\mathrm{p}_{\partial K^{r, t}}$ onto $\partial K^{r, t}$ is then defined on the offset $U_{t}:=\left(\partial K^{r, t}\right)^{t}$. Since $\partial K^{r, t} \subset U_{t}$, the map $\mathrm{p}_{\partial K^{r, t}}$ induces a one-to-one map between $\partial K^{\prime r, t}$ and $\partial K^{r, t}$. We now define

$$
\begin{array}{rlc}
\psi: U_{t} \times \mathbb{R}^{d} & \rightarrow & \operatorname{spt}\left(N\left(K_{r, t}\right)\right) \\
(x, n) & \mapsto\left(\mathrm{p}_{\partial K^{r, t}}(x), n_{\mathrm{p}_{\partial K^{r, t}}(x)}\right)
\end{array} .
$$

Using the affine homotopy between $\psi$ and the identity, the homotopy Lemma [13, 4.1.9 page 363-364] allows to show that $N\left(K^{r, t}\right)$ and $N\left(K^{\prime r, t}\right)$ are close. More precisely

$$
N\left(K^{r, t}\right)-N\left(K^{\prime r, t}\right)=\partial R,
$$

where $\partial R$ is the boundary of a particular $d$-rectifiable $R$ current whose support $\operatorname{spt}(R)$ has a $d$-volume bounded by $\mathcal{H}^{d}(\operatorname{spt}(R)) \leq k(r, \mu, d) \mathcal{H}^{d-1}\left(\partial K^{\prime r, t}\right) \sqrt{\varepsilon}$, where $\mathcal{H}^{k}$ denotes the k-dimensional Hausdorff measure and $k(r, \mu, d)$ is a constant that only depends on $r, \mu$ and $d$.

\section{Step 3}

Here, instead of applying the measures to a Borel set, we apply them to a function $f: \mathbb{R}^{d} \rightarrow \mathbb{R}$ (that can be for example indicatrix of Borel sets). Let us take an differential form $\omega_{k}$ associated to a given curvature measure. We could also consider the form $\omega_{\mathrm{H}}^{X, Y}$ associated to the anisotropic curvature measure. By combining previous equations, one has:

$\Phi_{\overline{\mathbb{R}^{d} \backslash K^{r}}, k}(f)-\Phi_{\overline{\mathbb{R}^{d} \backslash K^{\prime r}}, k}(f)=N\left(\overline{\mathbb{R}^{d} \backslash K^{r}}\right)\left(\bar{f} \omega_{k}\right)-N\left(\overline{\mathbb{R}^{d} \backslash K^{\prime r}}\right)\left(\bar{f} \omega_{k}\right)=F_{-t \sharp} \partial R\left(\bar{f} \omega_{k}\right)$.

We show that $F_{-t}$ is $\sqrt{1+t^{2}}$-Lipschitz. Since $\omega_{k}$ and $\mathrm{d} \omega_{k}$ are uniformly bounded by a constant depending on the dimension, $\operatorname{Lip}(\bar{f})=\operatorname{Lip}(f)$, one gets by Stokes theorem:

$$
\left|\Phi_{\overline{\mathbb{R}^{d} \backslash K^{r}}, k}(f)-\Phi_{\overline{\mathbb{R}^{d} \backslash K^{\prime r}}, k}(f)\right| \leq k(r, \mu, d)\|f\|_{\mathrm{BL}} \mathcal{H}^{d-1}\left(\partial K^{\prime r, t}\right) \sqrt{\epsilon},
$$

The previous inequality still holds for $K^{r}$ and $K^{\prime r}$. To conclude the proof, we use the bound on $\mathcal{H}^{d-1}\left(\partial K^{\prime r, t}\right)$ [8] and also use the critical function stability result to get rid of the assumption on the $\mu$-reach of $K$.

\section{References}

[1] P. Alliez, D. Cohen-Steiner, Y. Tong, and M. Desbrun. Voronoi-based variational reconstruction of unoriented point sets. In Proceedings of the Eurographics Symposium on Geometry Processing, pages 39-48, 2007. 
[2] N. Amenta and M. Bern. Surface reconstruction by Voronoi filtering. Discrete and Computational Geometry, 22(4):481-504, 1999.

[3] Piermarco Cannarsa and Carlo Sinestrari. Semiconcave functions, Hamilton-Jacobi equations, and optimal control. Progress in Nonlinear Differential Equations and their Applications, 58. Birkhäuser Boston Inc., Boston, MA, 2004.

[4] Frédéric Chazal, David Cohen-Steiner, and André Lieutier. Normal cone approximation and offset shape isotopy. Computational Geometry, 42(6):566-581, 2009.

[5] Frédéric Chazal, David Cohen-Steiner, and André Lieutier. A sampling theory for compact sets in euclidean space. Discrete $\&$ Computational Geometry, 41(3):461-479, 2009.

[6] Frédéric Chazal, David Cohen-Steiner, André Lieutier, and Boris Thibert. Shape smoothing using double offsets. In Proceedings of the 2007 ACM symposium on Solid and physical modeling, pages 183-192. ACM, 2007.

[7] Frédéric Chazal, David Cohen-Steiner, André Lieutier, and Boris Thibert. Stability of curvature measures. In Computer Graphics Forum, volume 28, pages 1485-1496. Wiley Online Library, 2009.

[8] Frédéric Chazal, David Cohen-Steiner, and Quentin Mérigot. Boundary measures for geometric inference. Foundations of Computational Mathematics, 10(2):221-240, 2010.

[9] David Cohen-Steiner and Jean-Marie Morvan. Second fundamental measure of geometric sets and local approximation of curvatures. Journal of Differential Geometry, 74(3):363-394, 2006.

[10] Louis Cuel, Jacques-Olivier Lachaud, Quentin Mérigot, and Boris Thibert. Robust geometry estimation using the generalized voronoi covariance measure. arXiv preprint arXiv:1408.6210, 2014.

[11] Herbert Edelsbrunner. The union of balls and its dual shape. In Proceedings of the ninth annual symposium on Computational geometry, pages 218-231. ACM, 1993.

[12] Herbert Federer. Curvature measures. Trans. Amer. Math. Soc., 93:418-491, 1959. 
[13] Herbert Federer. Geometric measure theory. Springer, 1969.

[14] J.H.G. Fu. Tubular neighborhoods in Euclidean spaces. Duke Math. J, 52(4):1025-1046, 1985.

[15] Joseph Fu. Tubular neighborhoods in euclidean spaces. Duke Mathematical Journal, 52(4):1025-1046, 1985.

[16] Daniel Hug, Markus Kiderlen, and Anne Marie Svane. Voronoi-based estimation of minkowski tensors from finite point samples. arXiv preprint arXiv:1511.02394, 2015.

[17] Daniel Hug and Rolf Schneider. Local tensor valuations. Geometric and Functional Analysis, 24(5):1516-1564, 2014.

[18] A. Lieutier. Any open bounded subset of $\mathbb{R}^{n}$ has the same homotopy type as its medial axis. Computer Aided Geometric Design, 36(11):1029-1046, 2004.

[19] Quentin Mérigot. Geometric structure detection in point clouds. Theses, Université Nice Sophia Antipolis, December 2009.

[20] Quentin Merigot, Maks Ovsjanikov, and Leonidas Guibas. Voronoibased curvature and feature estimation from point clouds. IEEE Transactions on Visualization and Computer Graphics, 17(6):743-756, 2011.

[21] Th Motzkin. Sur quelques propriétés caractéristiques des ensembles convexes. Atti Accad. Naz. Lincei Rend. Cl. Sci. Fis. Mat. Natur, $21: 562-567,1935$.

[22] A. Petrunin. Semiconcave functions in Alexandrov's geometry. In Surveys in differential geometry. Vol. XI, pages 137-201. Int. Press, Somerville, MA, 2007.

[23] Jan Rataj and Martina Zähle. Normal cycles of lipschitz manifolds by approximation with parallel sets. Differential Geometry and its Applications, 19(1):113-126, 2003.

[24] Jan Rataj and Martina Zähle. General normal cycles and lipschitz manifolds of bounded curvature. Annals of Global Analysis and Geometry, $27(2): 135-156,2005$.

[25] H. Weyl. On the volume of tubes. American Journal of Mathematics, 61(2):461-472, 1939 